\title{
ENSINO, PESQUISA E EXTENSÃO UNIVERSITÁRIA: EM BUSCA DE UMA INTEGRAÇÃO EFETIVA
}

\author{
Hedioneia Maria Foletto Pivetta* \\ Dirce Stein Backes ** \\ Adriana Carpes ${ }^{* * *}$ \\ Amara Lúcia Holanda Tavares Battistel ${ }^{* * * *}$ \\ Mara Marchiori*****
}

\section{Resumo}

Assumir uma atitude inovadora e transformadora da realidade social implica ampliar e fortalecer a integração entre ensino, pesquisa e extensão, enquanto processo formativo sistêmico. Estes escritos têm por objetivo refletir e discutir sobre a integração entre ensino, pesquisa e extensão na formação de profissionais da saúde, por meio de um estudo de caso que explicita como esse processo vem sendo realizado. A abordagem utilizada é qualitativa, com característica de observação participante. Assim, considerando o universo e a complexidade da formação humana, pressupõese a necessidade de viabilizar aprendizagens significativas, produzidas a partir da aquisição do conhecimento socialmente elaborado e contextualizado. Formação essa capaz de proporcionar ao profissional intervenções na e sobre a realidade, construídas com autonomia e competência para um fazer vinculado à prática social, geradora de novos saberes e novos fazeres viabilizados pela integração entre ensino, pesquisa e extensão.

Palavras-chave: Educação superior. Integração ensino-pesquisa-extensão universitária. Interdisciplinaridade. Responsabilidade social.

* Fisioterapeuta. Doutoranda e Mestre em Educação pela UFSM. Docente do Curso de Fisioterapia da Unifra. Membro do Grupo de Pesquisa Gipes e do Grupo Trajetórias de Formação (Gtforma) (hedioneia@unifra.br).

** Enfermeira. Doutora em Filosofia da Enfermagem. Docente do Curso de Enfermagem da Unifra. Membro do Grupo de Estudos e Pesquisas em Administração de Enfermagem e Saúde (Gepades UFSC) e Líder do Grupo de Estudos e Pesquisa em Empreendedorismo Social da Enfermagem e Saúde (Gepeses) (backesdirce@ig.com.br).

*** Farmacêutica e Bioquímica. Doutora em Química Orgânica. Docente do Curso de Farmácia do Centro Universitário Franciscano (Unifra), Santa Maria, Rio Grande do Sul, Brasil. Membro do Grupo Interdisciplinar de Pesquisa em Saúde (Gipes) (carpes.ad@gmail.com).

**** Terapeuta Ocupacional. Mestre em Educação. Docente do Curso de Terapia Ocupacional da Unifra, Santa Maria, Rio Grande do Sul, Brasil (amara@unifra.br).

${ }^{* * * * *}$ Enfermeira. Mestre em Educação. Docente do Curso de Enfermagem da Unifra. Membro do Gepeses (mara.marc@hotmail.com). 


\section{Introdução}

Entre os diferentes espaços de construção do conhecimento, a universidade ocupa um lugar privilegiado de convivência e desenvolvimento humano, científico-tecnológico e social. Tem como eixo central a formação de profissionais-cidadãos, isto é, de profissionais comprometidos com o desenvolvimento social em nível local e global.

Pensar as funções da universidade hoje, pautadas em princípios democráticos e transformadores, implica adentrar novos paradigmas que possibilitam olhares ampliados, além do diálogo entre os diferentes saberes disciplinares e a integração entre ensino, pesquisa e extensão.

O artigo 207 da Constituição Brasileira, ao contemplar essa integração, trouxe à tona uma proposta inovadora e, ao mesmo tempo, desafiadora para as universidades em geral (BRASIL, 1988). O artigo em questão convoca à reflexão para que as universidades gerem atividades de ensino, pesquisa e extensão de modo integrativo e complementar, promovendo a difusão, criação, sistematização e transformação do conhecimento por meio da articulação entre teoria e prática.

Nessa perspectiva, forma-se um ciclo dinâmico e interativo em que a pesquisa aprimora e produz novos conhecimentos, os quais são difundidos por meio do ensino e da extensão, fazendo com que esses três pilares balizadores da formação universitária tornem-se complementares e interdependentes, atuando de forma sistêmica.

O ensino, a pesquisa e a extensão, enquanto atividades complementares e interdependentes, precisam ter valorações equivalentes no sistema universitário, sob o risco de desenvolver conhecimento mutilante e reducionista. A qualidade e o sucesso dos profissionais formados pelas universidades dependem, em grande parte, do nível de interação e articulação entre esses três pilares do conhecimento uno e multidimensional. É difícil, portanto, conceber um aluno universitário bem sucedido sem a influência de uma formação sistêmica, isto é, ampliada e integrada, propiciada pelo ensino, a pesquisa e a extensão (FÓRUM DE PRÓ-REITORES DE EXTENSÃO DAS UNIVERSIDADES PÚBLICAS BRASILEIRAS, 2006).

O princípio da integração entre os pilares em questão reflete um conceito de qualidade do desempenho acadêmico capaz de favorecer a auto-reflexão crítica, a emancipação teórico-prática e o significado de responsabilidade social proporcionado pela aproximação entre a universidade e a comunidade. A concretização desse princípio supõe, portanto, a realização de projetos coletivos inseridos na comunidade e a integração dos diferentes saberes profissionais para a apreensão dos problemas de forma ampla, efetiva e resolutiva (MORIN, 2000). 
A integração entre o ensino, a pesquisa e a extensão transcende, a partir da compreensão sistêmica, a noção tecnicista e biologicista de competência profissional sustentada pelo saber tradicional reducionista (MORIN, 2004, 2007). A noção de competência profissional, pelo olhar sistêmico integrador e articulador, envolve o domínio não apenas do conhecimento acumulado e suas aplicações pontuais imediatas, mas também o processo de contextualização de como este conhecimento é produzido, sistematizado e empreendido no sentido de possibilitar a transformação social.

Mais especificamente na área da saúde, estimulados pelas novas Diretrizes Curriculares Nacionais, os estudantes têm a sua inserção na comunidade já a partir dos primeiros semestres da graduação. Essa inserção precoce na comunidade vem possibilitando a formação de um profissional mais crítico, criativo e sensível à problemática social. Percebe-se, nesse sentido, que novas abordagens de ensino-aprendizagem vêm ganhando espaço, pois as práticas tradicionais assistencialistas e distantes da realidade social são caracterizadas por uma sequência de ações padronizadas, cujo foco está centrado na disseminação de informações e no cumprimento de conteúdos programáticos (BACKES et al., 2007; MENDES, 2007).

Esse processo, atendendo às novas Diretrizes Curriculares Nacionais e aos Princípios formativos do Sistema Único de Saúde (SUS), vem crescentemente sendo focado e busca adotar metodologias participativas e problematizadoras, com o propósito de gerar práticas inovadoras e pró-ativas. Estas, por sua vez, procuram, além da formação profissional, mudanças comportamentais coerentes com os novos processos interdisciplinares de atendimento às necessidades dos indivíduos. Esse pensar vem se estruturando pela ênfase voltada à formação de atitudes, habilidades e competências humano-interativas capazes de responder às necessidades sociais emergentes. Definida como instituição de ensino superior pluricurricular e reconhecida pela propagação da filosofia franciscana de reverência à vida e a tudo que a cerca, a Instituição está empenhada em desenvolver a sua função de maneira comprometida e socialmente responsável, por meio das diferentes áreas do conhecimento.

Essa iniciativa vem sendo contemplada por meio de ações extensionistas das práticas de ensino, desenvolvidas junto ao entorno social, as quais fomentam novos projetos de extensão e pesquisa. A experiência, apesar das lacunas ainda existentes, tem demonstrado que é possível desenvolver uma formação universitária sistêmica por meio da integração entre o ensino, a pesquisa e a extensão, que interagem como via de mão dupla. Enquanto a universidade leva o conhecimento e a assistência à comunidade por meio de atividades de ensino e de extensão, estas se constituem como fontes de 
pesquisas que, por sua vez, retroalimentam o ensino. Assim, o ensino e a extensão são geradores de novas pesquisas, na medida em que identificam necessidades, anseios, aspirações e o saber que lhes é próprio. Portanto, tendo por premissa as reflexões anteriores, surgem as seguintes indagações: É possível realizar uma formação sólida sem inter-relacionar o ensino, a pesquisa e a extensão universitária? Como proporcionar aprendizagens significativas? Como está acontecendo a formação a partir da Reforma Sanitária Brasileira e das novas Diretrizes Curriculares Nacionais?

A partir do exposto, o presente texto tem por objetivo refletir e discutir sobre a integração entre ensino, pesquisa e extensão, por meio de um estudo de caso de como esse processo vem sendo concebido nos cursos da área da saúde de uma instituição confessional do interior do Estado do Rio Grande do Sul. Organiza-se a partir dos pressupostos teóricos da Reforma Sanitária Brasileira e a integração entre ensino, pesquisa e extensão, da Educação Superior como um universo formativo em transformação e do estudo de caso em si.

A metodologia utilizada é de cunho qualitativo e se caracteriza como observação participante, uma vez que traz a vivência de docentes em suas atividades de ensino, pesquisa e extensão com o olhar sistêmico e integrador.

\section{Compreendendo a integração entre ensino, pesquisa e extensão pelo olhar da Reforma Sanitária Brasileira}

O processo formativo dos profissionais da saúde, assim como a formação das demais áreas, não pode mais estar desvinculado dos mecanismos da globalização, bem como das políticas de saúde e educação vigentes.

Nesse sentido, é pertinente trazer à discussão a temática da Reforma Sanitária Brasileira, que culminou com a proposição formativa do SUS. Este, por sua vez, demandou a necessidade de uma revisão do processo formativo para os profissionais da área da saúde. As tentativas de implementação do SUS deixaram evidências de uma rediscussão acerca da qualidade, efetividade e resolutividade das ações de saúde. Surge, nessa perspectiva, a necessidade de ampliar e estreitar as relações entre os Ministérios da Saúde e da Educação, com a finalidade de traçar metas que contemplem a integralidade do indivíduo em seu contexto real e concreto. Para implementar as referidas mudanças no processo formativo dos diferentes cursos da área da saúde, foi necessária, então, a proposição de novas Diretrizes Curriculares Nacionais (BRASIL, 2001a) e a criação de novas estratégias curriculares, capazes de viabilizar a articulação dos princípios e diretrizes imprescindíveis ao processo formativo desse novo perfil profissional. 
Há um consenso de que a educação superior precisa priorizar uma formação para além da técnica, ou seja, pela compreensão do ser humano como um ser integral e integrador, autor e protagonista da sua própria história. O conhecimento técnico-científico se constitui como uma das vertentes ou dimensões do processo formativo. Logo, a formação engloba aspectos de produção de subjetividade, produção de habilidades técnico-interativas capazes de integrar e articular a complexa rede de interações sociais.

Considerando que a contemporaneidade exige um novo perfil de profissional, que seja capaz de transcender os preceitos normativo-reducionistas, subentende-se que essa formação demanda novas relações e interações pedagógicas, bem como novos processos de ensino-aprendizagem. Sob esse enfoque, emerge a reflexão acerca da integração entre as atividades de ensino, pesquisa e extensão universitária, como bem expressa o autor:

A mobilização do setor de saúde com a definição das Diretrizes Curriculares Nacionais correspondeu à preocupação com a consolidação do SUS, mas também correspondeu ao esforço intelectual de romper definitivamente com o paradigma biologicista, medicalizante, hospitalocêntrico e procedimentocentrado, atendendo aos novos desafios da contemporaneidade na produção de conhecimento e na construção das profissões (PINHEIRO, 2006, p. 21).

No sentido de romper com o paradigma biologicista, é mister criar condições sólidas no cotidiano acadêmico, proporcionando aprendizagem significativa e de valoração do indivíduo como um ser integral; assim, o profissional estará apto a atuar no mundo da vida e pela vida. Pela via das atividades extensionistas, o acadêmico tem a oportunidade de vivenciar in loco as concepções difundidas pelo conhecimento científico construído e acumulado pela humanidade, perpassado pelos processos de ensino e pesquisa. Ao mesmo tempo, levar o conhecimento à sociedade pode proporcionar a inserção na realidade local, possibilitando uma visão crítica do contexto sociocultural, bem como uma interconectividade entre a teoria e a prática, o que certamente contribuirá para o desenvolvimento de um sujeito autônomo e comprometido com o bem-estar da coletividade. E, ainda, espera-se que esse movimento possa gerar a produção de conhecimento consistente, coerente e condizente com a realidade e necessidades de saúde da comunidade assistida.

Para alcançar esse perfil na formação dos profissionais da saúde, as novas diretrizes curriculares estabelecem como objetivo, de acordo com o Parecer CNE/CES 1.300:

[...] levar os alunos dos cursos de graduação em saúde a aprender a aprender que engloba aprender a ser, aprender a fazer, aprender a viver juntos e aprender a conhecer, garantindo a capacitação de profissionais com autonomia e 
discernimento para assegurar a integralidade da atenção e a qualidade e humanização do atendimento prestado aos indivíduos, famílias e comunidades (BRASIL, 2001b, p. 4).

Considerando o objetivo das novas diretrizes curriculares, salienta-se que:

Aprender a conhecer como estratégia a mobilizar a descoberta e construção de novos conhecimentos, seja este científico e/ ou da realidade em que se está inserido, do contexto sócio-econômico-cultural em que se desenvolvem as ações em saúde; este pilar nos remete à pesquisa;

Aprender a fazer nos diz da competência técnica da atuação profissional, a qual não pode deixar de estar atrelada ao conhecimento da realidade do território e das relações interpessoais estabelecidas entre profissional/usuário/família/equipe/gestão. Tal competência se constrói no fazer cotidiano, nas ações práticas que nos remetem às atividades extensionistas;

Aprender a viver junto, saindo do seu lugar confortável e confiável para o encontro com o outro, no espaço alheio, aprendendo a conviver com a comunidade, estar com o outro, ou seja, a transposição dos conhecimentos teóricos para a vida prática;

Aprender a ser, incorporando ao processo formativo a dimensão humana, alia-se o conhecimento técnico ao ético, crítico, criativo e espiritual, o que vem a refletir sobre a responsabilidade social, autonomia e rigor científico; estes pressupostos provêm da articulação das atividades formativas orientadas pelo ensino, pesquisa e extensão.

Assim, considerando o universo e a complexidade da formação humana a que se propõe a universidade, pressupõe-se a necessidade de viabilizar aprendizagens significativas, produzidas a partir da aquisição do conhecimento socialmente elaborado e contextualizado, capaz de realizar intervenções na e sobre a realidade, construídas com autonomia e competência para um fazer vinculado à prática social, geradora de novos saberes e novos fazeres.

\section{Educação superior: um universo em transformação}

As sociedades contemporâneas são frequentemente descritas como sociedades do conhecimento. As atividades econômicas, sociais, culturais e outras atividades humanas tornaram-se dependentes de um enorme volume de conhecimento e informação. Mas a importância desse conhecimento não pode ser mensurada somente pelo número de profissionais habilitados que adentram no mercado de trabalho, mas principalmente pelo impacto que a formação deve apresentar sobre o crescimento intelectual e pessoal desse profissional, bem como o benefício que este pode representar para a sociedade. 
Tradicionalmente, as instituições de educação superior preocupavam-se com a excelência técnica na formação, em detrimento de outros aspectos não menos importantes, como o humanístico, ético, crítico-reflexivo e criativo. Nessa estruturação, a teoria era apresentada sempre antes da prática, e esta última deveria ser compreendida como uma aplicação da primeira. Assim, o conhecimento era trabalhado muito mais como produto do que como processo (LÁZARO, 1999).

A universidade, mais especificamente, originou-se de faculdades individualizadas, cuja principal função era a de formar profissionais (MOROZ, 2001). E, dessa forma, desenvolveu-se sem questionar que tipo de profissional deveria formar, tomando uma direção com ênfase no conhecimento fragmentado. Atualmente, porém, as sociedades atravessam um período de intensas mudanças, com o questionamento de inúmeros paradigmas que, ao longo do século XX, redirecionaram seu desenvolvimento. Os desafios que a complexidade da sociedade atual impõe requerem a implementação de uma educação transformadora, com respostas igualmente complexas.

A regulamentação da nova Lei de Diretrizes e Bases da Educação (LDB) (BRASIL, 1996) estabeleceu profundas mudanças nas estruturas curriculares das universidades, destacando-se pela extinção do currículo mínimo, maior autonomia e flexibilidade curricular, além da admissão de conhecimentos fora dos padrões da escola formal. O pressuposto essencial do novo paradigma é uma prática pedagógica que possibilite a produção do conhecimento e que permita um encontro de abordagens e tendências pedagógicas que atendam às exigências da sociedade.

O desafio dessas mudanças substanciais na virada do século, e em especial do paradigma da ciência, levou ao repensar do sistema educacional como um todo e, nesse contexto, a educação superior é considerada um componente essencial, tendo em vista os novos desafios com os quais passou a se confrontar. No preâmbulo da declaração emitida durante a Conferência Mundial de Educação Superior, realizada pela Unesco, em Paris (1999), foram feitas as seguintes considerações:

No limiar de um novo século, há uma demanda sem precedentes e uma grande diversificação na educação superior, bem como maior consciência sobre a sua importância vital tanto para o desenvolvimento sociocultural e econômico, como para a construção do futuro, diante do qual as novas gerações deverão estar preparadas com novas habilitações, conhecimentos e ideais (CONFERÊNCIA MUNDIAL SOBRE O ENSINO SUPERIOR, 1999, p. 17).

Esse movimento de mudança se estendeu não somente à formação discente, mas também ao corpo docente, do qual se exigiu um novo papel de 
articulador e mediador entre o conhecimento elaborado e o conhecimento a ser produzido (BENHRENS, 1999). Nesse contexto, entende-se que, mais do que dispositivos legais, faz-se necessário o entendimento da dimensão dessas mudanças e a implicação de todos os envolvidos nesse processo, principalmente dos professores, responsáveis pela concretização da proposta.

A publicação das Diretrizes Curriculares Nacionais (DCN) (BRASIL, 2001a) foi um importante marco histórico na formação dos profissionais da saúde, ao determinar como foco a preparação desse profissional para atuar no Sistema Único de Saúde (SUS), articulado ao contexto social, participando e contribuindo para o desenvolvimento da sociedade.

Dessa maneira, o processo de formação dos profissionais da saúde, antes centrado na doença, busca construir uma abordagem integral do indivíduo no processo saúde-doença, tendo por princípio a ênfase na atenção básica. Com isso, as mudanças na graduação deverão resultar na formação de profissionais com atitudes, competências e habilidades para atuar com qualidade, eficiência e resolutividade no SUS, considerando o processo da Reforma Sanitária Brasileira, visando com isso uma formação mais pró-ativa, isto é, focada na promoção, proteção, recuperação e reabilitação da saúde (BRASIL, 2006).

\section{Dinâmica de construção da integração entre ensino, pesquisa e extensão: 0 estudo de caso}

Com o propósito de se integrar e comprometer-se com os avanços e políticas delineadas para a formação dos profissionais da saúde, vêm-se implementando modificações contínuas nas matrizes curriculares dos diferentes cursos da área da saúde, compreendendo que a estrutura curricular e a organização pedagógica precisam adequar-se às necessidades e prioridades da formação para os profissionais da saúde no País.

Salienta-se que essas modificações estão presentes de maneira bastante efetiva nas atividades teórico-práticas da formação profissional dos cursos da saúde de uma Instituição de Ensino Superior (IES) Confessional do interior do Estado do Rio Grande do Sul. Assim, os projetos pedagógicos dos cursos mostram-se cada vez mais articulados com o Projeto Pedagógico Institucional (PPI) (UNIFRA, 2007a), conforme exposto a seguir:

[...] estruturação dos cursos de graduação e pós-graduação se dá de forma a promover profissionais comprometidos com a realidade sócio-cultural, conjugando o senso reflexivo ao crítico-criativo, com vistas à apropriação e reelaboração e produção do saber, no avanço da ciência e do conhecimento da realidade (UNIFRA, 2007a, p. 2). 
[...] Concebe-se o conhecimento como compreensão da realidade em que se vive cuja apreensão ocorre no processo reflexão-ação. Dessa forma, o saber construído na experiência propicia ao ser humano condições de responder às exigências do tempo e do espaço em que está inserido (UNIFRA, 2007a, p. 32).

Assim, os cursos da área da saúde buscam novos cenários de práticas e estimulam a inserção precoce dos acadêmicos nos diferentes contextos sociais e em diferentes níveis de intervenção, possibilitando a articulação da teoria com a prática desde os primeiros semestres de formação acadêmica. Acredita-se que, dessa maneira, por meio de atividades de ensino propicia-se a extensão universitária junto à comunidade, o que gera uma formação mais contextualizada, crítica e reflexiva, pautada nos princípios do sistema de saúde vigente em nosso país.

Em nível de atenção básica, surgiu, por meio da Pró-Reitoria de Extensão (PROEX) da referida IES, a iniciativa de estabelecer as práticas formativas da área da saúde junto a uma região do município. Assim, desde o ano de 2004, no intuito de fortalecer e ampliar as ações de saúde, realiza-se o Projeto, que posteriormente transformou-se em Programa, Atenção Integral a Saúde e a Qualidade de Vida. Essa iniciativa estimulou e convocou, também, a participação de outras áreas de conhecimento, como das ciências humanas, a fim de assegurar a efetividade e resolutividade das ações de saúde. O Programa em questão, que abriga outros grandes projetos institucionais, partiu da necessidade de focar e estimular as práticas interdisciplinares de ensino, pesquisa e extensão, no intuito de gerar maior impacto das ações de saúde no campo de prática social.

No contexto do Programa Atenção Integral a Saúde e a Qualidade de Vida destacam-se projetos como: O construir interdisciplinar e a prevenção à violência contra a criança e o adolescente; Saúde na escola com foco em educação e promoção da saúde; Abordagem interdisciplinar no cuidado do usuário portador de doenças crônicas; Tecnologias para a sustentabilidade da água em zonas rurais; Trabalho de inclusão social com catadores de material reciclável, entre outros. Recentemente, a instituição foi contemplada, também, com o "Pró-Saúde", caracterizado como um Programa Nacional de Reorientação da Formação Profissional em Saúde (BRASIL, 2007), focado na formação de recursos humanos, produção de conhecimentos e prestação de serviços de saúde focados no SUS.

A ideia de centralizar as práticas/ações de saúde da IES, nessa região mais especificamente, teve o intuito de promover ações interdisciplinares pela interatividade e integralidade da atenção humanizada, o trabalho em equipe e o fomento de parcerias entre ensino e os serviços de saúde, bem como 
estreitar os vínculos e favorecer a continuidade do cuidado em saúde. Nessa ótica, o ensino perpassa a extensão, assim como a extensão perpassa o ensino e a pesquisa, pois possibilita um processo formativo ativo e "extramuros", isto é, comprometido com o ser humano em seu contexto real e concreto.

As diversas atividades de ensino-aprendizagem, em nível de atenção básica, ocorrem por meio de visitas e cuidados domiciliares, atendimentos e orientações individuais e coletivas de promoção e proteção da saúde, bem como a prevenção de doenças, como o combate à dengue, o programa HiperDia (BRASIL, 2002, 2006), os Mutirões de Saúde, Assistência e Atenção Farmacêutica, discussão de casos clínicos, entre outros.

A relação do ensino com os serviços de saúde também se estende a outros cenários, além das Unidades de Estratégia de Saúde da Família e Unidades Básicas de Saúde - UBS, como em organizações não governamentais, Farmácia Municipal de Saúde, Farmácia Popular do Brasil, Vigilância Epidemiológica, Centro de Diagnóstico e Atenção Secundária a Saúde, Hospitais e outros.

A Instituição também conta com atividades de ensino, pesquisa e extensão no Serviço Integrado em Saúde, que se constitui em um espaço para o ensino da prática profissional e compromisso social no contexto da própria Instituição. Segundo dados do Relatório de Gestão do Programa Atenção Integral a Saúde e a Qualidade de Vida da Região Oeste de Santa Maria (UNIFRA, 2007b), em conjunto com as ações do Serviço Integrado em Saúde, as atividades interdisciplinares realizadas em 2007 culminaram no atendimento de 15.043 pessoas. Nesse montante, envolveram-se 11 cursos de graduação, 89 docentes, 29 bolsistas, 50 acadêmicos voluntários, cerca de 500 alunos de graduação, três técnicos administrativos e 30 profissionais de instituições afins.

Como surge a pesquisa nesse cenário? A pesquisa constitui, nessa perspectiva, uma ferramenta indispensável para a geração de novas hipóteses, enraizadas e fundamentadas na vida e nas reais necessidades da população. As atividades formativas proporcionadas pelo ensino e por meio da extensão são geradoras de novos questionamentos e interpelações, logo, indutoras de novas hipóteses e práticas sociais pró-ativas. Esse processo formativo pode ser vislumbrado, de forma mais concreta, por meio dos Trabalhos de Final de Graduação (TFGs), dos Projetos de Iniciação Científica (PROBIC), dos Projetos de Extensão (PROEX), dentre outros.

A inserção da extensão, nos Planos Pedagógicos dos Cursos de Graduação, mais especificamente nas práticas acadêmicas de ensino, pesquisa e extensão, passou a ser objeto de reflexão e discussão crescente no âmbito das Direções de Área e nas Coordenações de Cursos, que buscaram constituir Grupos de Extensão formados por docentes e discentes, articulados em torno de um fim comum, cujo foco são as atividades de ensino e pesquisa ligadas às demandas 
sociais e às vivências da comunidade. Distante de constituir-se apenas como uma atividade assistencialista, as Linhas de Extensão são derivadas desses Grupos e caracterizam-se por apresentarem e articularem interesses investigativos de uma ou mais áreas de conhecimento, a fim de alimentar e retroalimentar o processo de pesquisa, mantendo a dialética de constante ebulição entre teoria - produção de conhecimento e prática - e aplicação do conhecimento.

As políticas de extensão da referida IES foram definidas a partir do entendimento de que a extensão universitária é o compromisso que a prática acadêmica assume ao aproximar e reaproximar a universidade, pelas atividades de ensino e de pesquisa, das demandas da sociedade, contribuindo para a formação de um sujeito íntegro e comprometido com a transformação social. É o papel da extensão universitária estimular e ampliar a rede de interações comunitárias, de tal modo que aquilo que se produz em termos de conhecimentos novos e aquilo que se difunde por meio do ensino não fiquem restritos ao espaço da sala de aula ou dos laboratórios, mas que encontrem aderência na realidade e para a realidade social.

Nessa perspectiva, a extensão universitária vai muito além da transmissão, divulgação ou aplicação do conhecimento pelo conhecimento. Busca, continuamente, problematizar o ensino pela vivência presencial, solidária e transformadora, e a partir daí constituir-se como geradora de pesquisa. O campo de extensão é expresso na prática acadêmica, na qual conflui a interação universidade-sociedade-universidade, por meio da articulação de atividades de ensino e pesquisa, articuladas com as demandas sociais emergentes, pela vivência de ser com a comunidade e de estar e sentir na comunidade.

O produto dessas práticas, que resulta na produção de novas tecnologias de cuidado em saúde e, principalmente, no engajamento responsável de docentes e discentes nas chamadas demandas sociais emergentes, permite argumentar que é possível vislumbrar um novo perfil formativo. Uma formação na qual a sociedade assume papel relevante, na qual o ser humano é autor e protagonista da própria história. Uma formação humana, solidária, alimentada pela rede de relações, interações e integrações sociais.

\section{Reflexões finais}

O desenvolvimento de competências profissionais interativas, associativas e socialmente responsáveis, implica necessariamente um conhecimento capaz de articular o ensino, a pesquisa e a extensão. A formação de competências profissionais para fazer frente à complexidade dos problemas sociais emergentes, implica um processo de aprendizagem que perpassa pela inserção na 
realidade social. Implica atitudes reflexivas e questionadoras que decorrem da interação com a comunidade, a fim de apreender as reais necessidades e, a partir destas, protagonizar ações pró-ativas e transformadoras. Acredita-se que, dessa maneira, criam-se condições para que a formação profissional transcenda os aspectos técnicos, instrumentais ou meramente teóricos, passando à contextualização a partir de uma visão ampliada, proporcionada pela integração dos aspectos técnico-científicos, éticos, políticos, econômicos e sócio-culturais.

A Instituição de Ensino Superior precisa vislumbrar, em suma, a formação integral a partir da estimulação e integração de novas metodologias de ensino-apredizagem, as quais direcionam e se agregam às Diretrizes da formação acadêmica, demandadas pelo Ministério da Saúde e Educação. Assim, constrói-se um saber-fazer que transcende o instrumental, integrando aspectos cognitivo-afetivos e comportamentais para o cuidado em saúde, na promoção da cidadania e, consequentemente, para o bem-estar social e a melhoria da qualidade de vida dos indivíduos e comunidades.

Assim, acredita-se que esse perfil formativo, conforme a experiência delineada, pode ser conquistada por meio da integração entre ensino, pesquisa e extensão universitária. Transcendendo o discurso e usufruindo de uma prática real, contextualizada e interdisciplinar, vislumbram-se, efetivamente, processos de ensino-aprendizagem geradores de novos saberes e novos fazeres em saúde.

\section{Referências}

BACKES, Dirce Stein et al. The practice of teaching and learning about nursing management based on Freire's methodology. Online Brazilian Journal of Nursing, Niterói, v. 6, n. 1, p. 45-57, 2007.

BENHRENS, Marilda Aparecida. A prática pedagógica e o desafio do paradigma emergente. Revista Brasileira de Estudos Pedagógicos, Brasília, v. 80, n. 196, p. 383-403, 1999. BRASIL. Constituição (1988). Constituição da República Federativa do Brasil. Belém: Basa, 1988. . Lei n ${ }^{\circ}$ 9.394, de 20 de dezembro de 1996. Estabelece as Diretrizes e Bases da Educação Nacional. Diário Oficial da República Federativa do Brasil, Brasília, DF, n. 248, 23 dez. 1996.

- Ministério da Educação. Conselho Nacional de Educação. Resolução CNE / CES n. 583, de 3 de abril de 2001. Diário Oficial da União, Brasília, DF, 2001a. Disponível em: <http:/ / www.mec.gov.br/cne>. Acesso em: 24 jan. 2009.

- Ministério da Saúde. Ministério da Educação. Programa Nacional de Reorientação da Formação Profissional em Saúde: Pró-Saúde. Brasília, DF: Ministério da Saúde, 2007. (Série C. Projetos, Programas e Relatórios). 
BRASIL. Ministério da Saúde. Conselho Nacional de Educação. Diretrizes Curriculares Nacionais dos Cursos de Graduação em Farmácia e Odontologia. Parecer CNE/CES n. 1300/01. Diário Oficial da União, Brasília, DF, seção 1, p. 25. 2001b.

. Ministério da Saúde. Secretaria de Políticas de Saúde. Plano de reorganização da atenção à hipertensão arterial e ao diabetes mellitus. Brasília: Ministério da Saúde; 2002. . Ministério da Saúde. Secretaria de Vigilância em Saúde. Política Nacional de Promoção da Saúde. Brasília: Ministério da Saúde, 2006.

CONFERÊNCIA MUNDIAL SOBRE O ENSINO SUPERIOR, 1998, Paris. Tendências da educação superior para o século XXI. Brasília: Crub, 1999.

FÓRUM DE PRÓ-REITORES DE EXTENSÃO DAS UNIVERSIDADES PÚBLICAS BRASILEIRAS, 2006. Indissociabilidade ensino-pesquisa-extensão e a flexibilização curricular: uma visão da extensão. Porto Alegre: UFRGS; Brasília: MEC, 2006.

LÁZARO, André. Visão e ação: a universidade no século XXI. Rio de Janeiro: Eduerj, 1999.

MENDES, Isabel Amélia Costa; MARZIALE, Maria Helena Palucci. O caminho inovador e empreendedor da Revista Latino-Americana de Enfermagem e a Adoção de Política de Internacionalização. Revista Latino-Americana de Enfermagem, Ribeirão Preto, v. 15, n. 2, p. 185-186, mar. / abr. 2007.

MORIN, Edgar. A cabeça bem feita: reformar a reforma reforça o pensamento. 10. ed. Rio de Janeiro: Bertrand Brasil, 2004.

. Ciência com consciência. 10. ed. Rio de Janeiro: Bertrand Brasil, 2007.

. Os sete saberes necessários à educação do futuro. São Paulo: Cortez; Brasília:

Unesco, 2000.

MOROZ, Melania. Que profissional formar?: questão desafiadora para a universidade. In: SEVERINO, Antônio Joaquim; FAZENDA, Ivani Catarina A. Conhecimento, pesquisa e educação. Campinas: Papirus, 2001. p. 67-79.

PINHEIRO, Roseni (Org.). Ensinar saúde: a integralidade e o SUS nos cursos de graduação na área da saúde. 2. ed. Rio de Janeiro: IMS, 2006.

UNIFRA. Projeto pedagógico institucional - PPI: res. 15/2007, de 27/06/07, do Conselho Universitário/Centro Universitário Franciscano. Santa Maria: Unifra, 2007a.

. Relatório anual do Proex: relatório da Pró-Reitoria de Extensão: Núcleo da Região Oeste de Santa Maria: Núcleo de Atenção Integral à Saúde e à Qualidade de Vida da Região Oeste de Santa Maria. Santa Maria: Unifra, 2007b. 


\section{Teaching, research and university extension: in search of an effective integration Abstract}

Taking an innovating and transforming attitude of the social reality implies expanding and strengthening the integration between teaching, research and extension as a systemic learning process. This paper is meant to reflect and discuss the integration between teaching, research and extension in the training of health professionals, by means of a case study that demonstrates how this process has been performed. The qualitative approach was performed through participating observation technique. Thus, considering the universe and the complexity of human development, the need to facilitate meaningful learning, produced from the acquisition of socially contextualized knowledge, is implied. This training makes it possible for the professional to intervene in and about the reality, through interventions made with autonomy and competence, generating new knowledge and new practices enabled by the integration of teaching, research and extension.

Keywords: Higher education. Integration of teaching, research and university extension. Interdisciplinarity. Social responsibility.

\section{Enseignement, recherche et extension universitaire : à la recherche d'une intégration effective \\ Résumé}

Assumer une attitude innovatrice et transformatrice de la réalité sociale implique amplifier et fortifier l'intégration entre enseignement, recherche et extension, en tant que processus formatif systémique. Ces écrits ont comme objectif réfléchir et discuter l'intégration entre enseignement, recherche et extension dans la formation des professionnels de la santé, par l'entremise d'une étude de cas qui explicite comment ce processus s'est réalisé. L'approche utilisée est qualitative avec une caractéristique d'observation participante. Ainsi, considérant l'univers et la complexité de la formation humaine, l'on présuppose la nécessité de viabiliser des apprentissages significatifs, produits à partir de l'acquisition du savoir socialement élaboré et contextuel. Cette formation est capable de proportionner au professionnel des interventions dans et sur la réalité, construites avec autonomie et compétence pour une action véhiculée à la pratique sociale, gérant de nouveaux savoirs viabilisés pour l'intégration entre enseignement, recherche et extension. Mots clefs : Enseignement supérieur. Intégration enseignement-recherche-extension universitaire, Interdisciplinarité. Responsabilité sociale.

\section{Enseñanza, investigación y extensión universitaria: en búsqueda de una integración efectiva \\ Resumen}

Asumir una actitud innovadora y transformadora de la realidad social implica ampliar y fortalecer la integración entre enseñanza, investigación y extensión, en cuanto proceso formativo sistémico. Estos escritos tienen por objetivo reflexionar y discutir sobre la integración entre enseñanza, investigación y extensión en la formación de profesionales de salud, por medio de un estudio de caso que explicita como se está realizando este proceso. El abordaje utilizado es cualitativo, con característica de observación participante. Así, considerando el universo y la complejidad de la formación humana, se presupone la necesidad de viabilizar aprendizajes significativos, producidos a partir de la adquisición del conocimiento socialmente elaborado y contextualizado. Formación esa capable de proporcionar al profesional intervenciones en y sobre la realidad, generadora de nuevos saberes y nuevos haceres viabilizados por la integración entre enseñanza, investigación y extensión.

Palabras clave: Educación Superior. Integración enseñanza/investigación/extensión universitaria. Interdisciplinaridad. Responsabilidad social.

Recebido em: 05.05.2010

Aceito em: 03.09.2010 